\title{
The influence of goals on sense of control
}

\author{
Wen Wen, Atsushi Yamashita, Hajime Asama \\ Department of Precision Engineering, University of Tokyo, 7-3-1 Hongo, Bunkyo-ku, \\ Tokyo 113-8656, Japan
}

Corresponding author:

Wen Wen

Department of Precision Engineering, University of Tokyo, 7-3-1 Hongo, Bunkyo-ku,

Tokyo 113-8656, Japan

Email: wen@ robot.t.u-tokyo.ac.jp

Telephone: +81-3-5841-6486; fax: +81-3-5841-8547 


\begin{abstract}
We examined the influence of goals on sense of control relative to that experienced when taking action randomly. In the experimental task, participants controlled the direction of a moving dot by pressing the left and right keys at will without a specific goal (the control condition), directed the moving dot to a destination as often as possible (the strong goal condition), or kept the moving dot in the central area of the screen (the weak goal condition) for as long as possible. The results showed that the strong goal impaired the sense of control, but the weak goal did not exert an influence. We concluded that the goal-based expectation influenced sense of control, but the goal-directed action selection did not. Furthermore, we proposed a modified comparator model of the sense of control, offering a promising approach to integration of the predictive and postdictive processes involved in the sense of control.
\end{abstract}

Keywords: sense of control, sense of agency, comparator model, goal, intention 


\section{Introduction}

In daily life, people send commands to objects, such as computers, cars, or ovens, to cause these machines work as intended. During such operation, a subjective feeling, "I am controlling it," emerges. This feeling is known as a sense of control (also called a sense of agency in the literature). Sense of control is important in explaining changes in the outside world and making and following decisions. However, the occurrence of events could match one's prediction or expectation coincidentally and induce an illusionary sense of control

According to the comparator model, a subjective feeling of control is produced via comparison of predicted and sensed information (Blakemore, Frith, \& Wolpert, 1999; Blakemore, Wolpert, \& Frith, 1998, 2002; Frith, Blakemore, \& Wolpert, 2000; Wolpert \& Flanagan, 2001; Wolpert \& Ghahramani, 2000). Specifically, a predicted state is generated via an efferent copy of a motor command and compared with the sensed state. The feeling of control emerges due to the match between the predicted and sensed states (Blakemore et al., 2002).

However, later research has suggested that predictive processes based on motor signals might not be necessary for the generation of a sense of control, which would also be influenced by contextual information and prior knowledge (Synofzik, Vosgerau, 
\& Newen, 2008; Wegner \& Wheatley, 1999). Wegner suggested that the sense of conscious will, including the sense of agency, could be an illusion based on postdictive inference (Wegner, 2002). In particular, when a thought occurs prior to an action (i.e., the priority principle), is consistent with that action (i.e., the consistency principle), and alternative causes do not exist (i.e., the exclusivity principle), people experience authorship of the action (Wegner, 2003). For example, Wegner and colleagues reported that participants felt an illusionary feeling of control over another's hand when they heard instructions for the other person's movements in advance (Wegner, Sparrow, \& Winerman, 2004). In their experiment, paired participants (i.e., helpers) stood behind participants with their arms stretched forward and performed a series of movements. The participants standing at the front could see themselves and the helpers' arms in a mirror. Although the participants did not perform any movements themselves, when they heard the instruction describing the helpers' movement in advance, they reported feeling a sense of agency over the actions performed by the helpers. This phenomenon matched the priority and consistency principle of Wegner's theory but did not contain motor signals. Therefore, it is often cited in studies suggesting that the comparator model does not account for the sense of control adequately. In recent research, most researchers in this field have agreed that both predictive and postdictive processes 
contribute to sense of control. For example, Synofzik and colleagues proposed a two-step account of agency, in which predictive processes are presented at a perceptual level, and postdictive processes are presented at a propositional level (Synofzik et al., 2008). Furthermore, Moore and Fletcher (2012) proposed a framework for cue integration, in which the authors suggested that multiple cues, both internal and external, contribute to the sense of agency, and the extent of this contribution depends on their reliability (Moore \& Fletcher, 2012).

In the present study, we focused on the influence of goals on the sense of control. In many daily situations, people take action to achieve specific goals as opposed to acting randomly. It is important to determine whether goals influence the sense of control in order to understand human behavior and verify the mechanism of the sense of agency. If the sense of control is relevant to a goal, when we actually use a machine to complete a job, we could find that the sense of control differs from that experienced in situations in which we used the machine without a specific goal.

We hypothesized that goals would influence sense of control. Our hypothesis had two foundations involving goal-directed intention and goal-based expectation. Pacherie categorized intention into three types: distal (D-intention), which refers to the intention to achieve a distal goal; proximal (P-intention), which refers to the will to 
initiate action; and motor (M-intention), which involves the motor representations underlying one's actions (Pacherie, 2008). When there is a goal present, people select optimal actions and timing to achieve the goal; therefore, distal and proximal intention are activated, form an expectation according to distal intention, and influence motor intention. In contrast, when there is no specific goal, the active levels of distal and proximal intention are at low levels, and only motor intention is dominantly involved in the development of the sense of control. In summary, if there is a goal present, both action selection and outcome expectation differ from those of conditions without a goal. The processes underlying action selection have been reported to affect the sense of control (Wenke, Fleming, \& Haggard, 2010); therefore, goal-directed motor intention could influence the sense of control. Furthermore, conscious expectation of a goal state has been reported to enhance experienced authorship (Aarts, Custers, \& Wegner, 2005); therefore, goal-based intention could also affect the sense of control. Moreover, when there is a specific goal present, people are likely to expect to achieve the goal and compare actual sensory feedback with their expectations. If the feedback does not match their expectations, the sense of control could be impaired (van der Weiden, Ruys, \& Aarts, 2013). Furthermore, a recent study reported that the comparison of motor commands and their effects would be uncertain when the effects were delayed, and in 
consequence, the comparison of goal and outcome would be more dominant relative to the action-effect comparison in such a condition (Wen, Yamashita, \& Asama, 2015).

In the present study, we examined the influence of goals on sense of control. In order to determine whether goal-directed action selection or goal-based expectation influence sense of control, the sense of control in two different goal conditions were compared with that of a condition without a goal (i.e., the control condition). Specifically, in the strong goal condition, a goal accompanied by performance feedback was provided for participants. In the weak goal condition, a similar goal was provided but no obvious state reflecting achievement of the goal was defined. If goal-directed action selection is important, sense of control in both the two goal conditions would differ from the one in the condition without a goal. If goal-based expectation alone is important, sense of control in the strong goal condition would differ from that of other conditions.

\section{Method}

\subsection{Participants}

A total of 20 students with normal or corrected-to-normal visual acuity participated in the experiment and received monetary compensation in return for their participation. Their mean age was 25.9 years $(S D=3.9$, range $22-32)$. The experiment 
was conducted according to the principles of the Helsinki Declaration and approved by the ethics committee of the Faculty of Engineering at the University of Tokyo. All participants provided written consent prior to participation.

\subsection{Stimuli and Task}

In each trial of the experimental tasks (Figure 1), a 5-mm black dot appeared in a random position on a $597 \mathrm{~mm} \times 336 \mathrm{~mm}$ (width $\times$ height) gray background, following the presentation of a 500-ms blank screen, and moved randomly at a speed of $124 \mathrm{~mm} / \mathrm{s}$. The direction of the dot did not change until it reached the borders of the screen or participants pressed the left or right key during the trial. When it reached the screen borders, the dot bounced back. Participants were instructed to press the left or right key on the keyboard to change the direction of the moving dot. The direction of the dot turned $20^{\circ}$ clockwise with a right key press and $20^{\circ}$ counterclockwise with a left key press and reflected a certain probability. The participants were told to press the left or right key repeatedly (the direction of the moving dot would not change if participants held the key down). There were four preset probabilities $(20 \%, 50 \%, 80 \%$, and $100 \%)$ that the moving dot would respond to the left or right key. This probability was consistent within each trial and varied randomly between trials. The moving dot disappeared from the screen after $10 \mathrm{~s}$, and participants rated the extent to which they 
felt that the dot had responded to their commands by clicking one of 11 radio buttons (to reflect $0-100 \%$ in $10 \%$ increments) using a mouse.

In the strong goal condition, a 16-mm empty square with a black border appeared at the center of the screen containing the moving dot. Participants were instructed to direct the dot into the square as quickly as possible. The square did not influence the motion of the dot (i.e., the moving dot would move through the square subsequent to arrival). The participants were instructed to direct the dot into the square again if the dot went though, as often as possible. In the weak goal condition, a cross appeared at the center of the screen. Participants were instructed to keep the moving dot in the central area (around the cross) for as long as possible. In the control condition, the moving dot was on the screen alone, and participants were instructed to press the left or right key randomly at will to confirm whether their commands were effective. In all three goal conditions, participants were instructed to form a judgment of control according to whether their commands were effective, without considering their performance. 
The strong goal condition
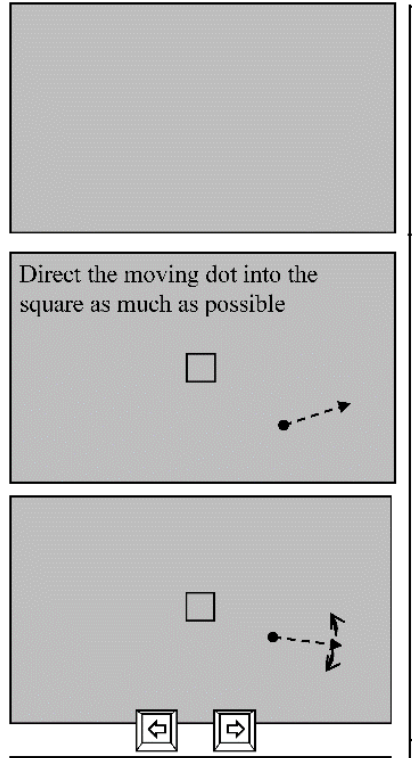

To what extent did the dot responded to your commands? $0 \%-100 \%$
The weak goal condition

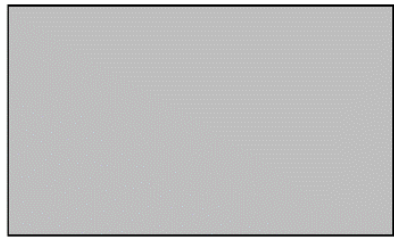

Keep the moving dot staying at the central area

$+$

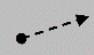

$10 \mathrm{~s}$

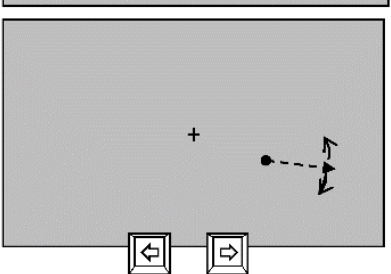

To what extent did the dot responded to your commands? $0 \%-100 \%$
The control condition

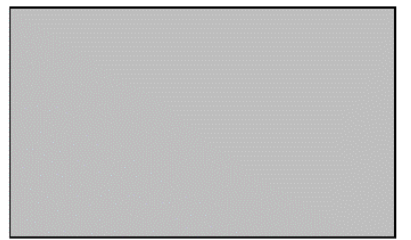

Press the left and right keys randomly as you wish
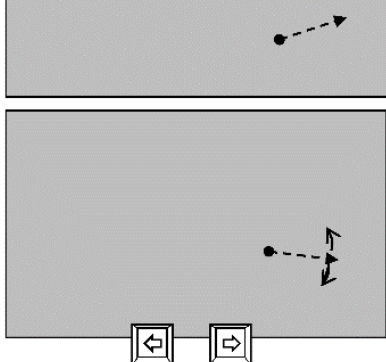

To what extent did the dot responded to your commands? $0 \%-100 \%$

Figure 1. The flow of each condition of the experimental task. Arrows with broken lines

indicate the direction in which the dot moved. Participants were instructed to direct the moving dot into the square as quickly as possible by pressing the left and right keys to turn the moving dot when they saw the square appear in the center of the screen (the strong goal condition), keep the moving dot in the central area (the weak goal condition), or press the left and right keys randomly at will (the control condition). Ten seconds after the appearance of the dot, participants used a mouse to rate the extent to which they felt that the dot had responded to their commands by providing a percentage between $0 \%$ and $100 \%$ (in increments of $10 \%$ ). 


\subsection{Procedure}

Participants were tested individually in a quiet room, seated on a chair positioned approximately $50 \mathrm{~cm}$ from a 27 -inch LCD monitor with a resolution of 1,920 $\times 1,080$ pixels $(0.311 \mathrm{~mm} /$ pixels $)$. Having received an explanation regarding the requirements of the experimental task, participants practiced for 10 random trials. Following the practice sessions, each participant complete 120 trials, comprising 10 trials involving each probability $(20 \%, 50 \%, 80 \%$, and 100\%) in each goal condition (strong goal, weak goal, and control condition), in random order. The 120 trials were divided into 3 blocks, each containing 40 trials. Participants took 5-min breaks between blocks. The experiment lasted for $50 \mathrm{~min}$ on average.

\section{Results}

The average ratings of control for each condition are shown in Figure 2. We conducted a 4 (effectiveness of control of $20 \%, 50 \%, 80 \%$, or $100 \%) \times 3$ (strong goal, weak goal, or control) repeated-measures ANOVA to compare rating scores. Unsurprisingly, the main effect of the effectiveness of control was significant $(F(3,57)$ $\left.=444.74, p<.01, \eta_{\mathrm{p}}{ }^{2}=0.96\right)$. The more effective the commands, the stronger sense of control the participants felt (according to Bonferroni multiple comparisons, differences between effectiveness conditions were all significant). More importantly, the main 
effect of goal was also significant $\left(F(2,38)=6.76, p<.01, \eta_{\mathrm{p}}{ }^{2}=0.26\right)$. Bonferroni multiple comparisons revealed that the rating in the strong goal condition significantly differed from that in the control condition $(p=.002)$, but the rating in the weak goal condition did not $(p=.237)$. The interaction between the effectiveness of commands and the goal was nonsignificant $\left(F(6,114)=1.04\right.$, n.s., $\left.\eta_{\mathrm{p}}{ }^{2}=0.05\right)$. In summary, the results revealed that only the existence of a goal accompanied by performance feedback impaired the sense of control. That is, the goal-based expectation strongly affected the sense of control but the goal-directed action selection did not.

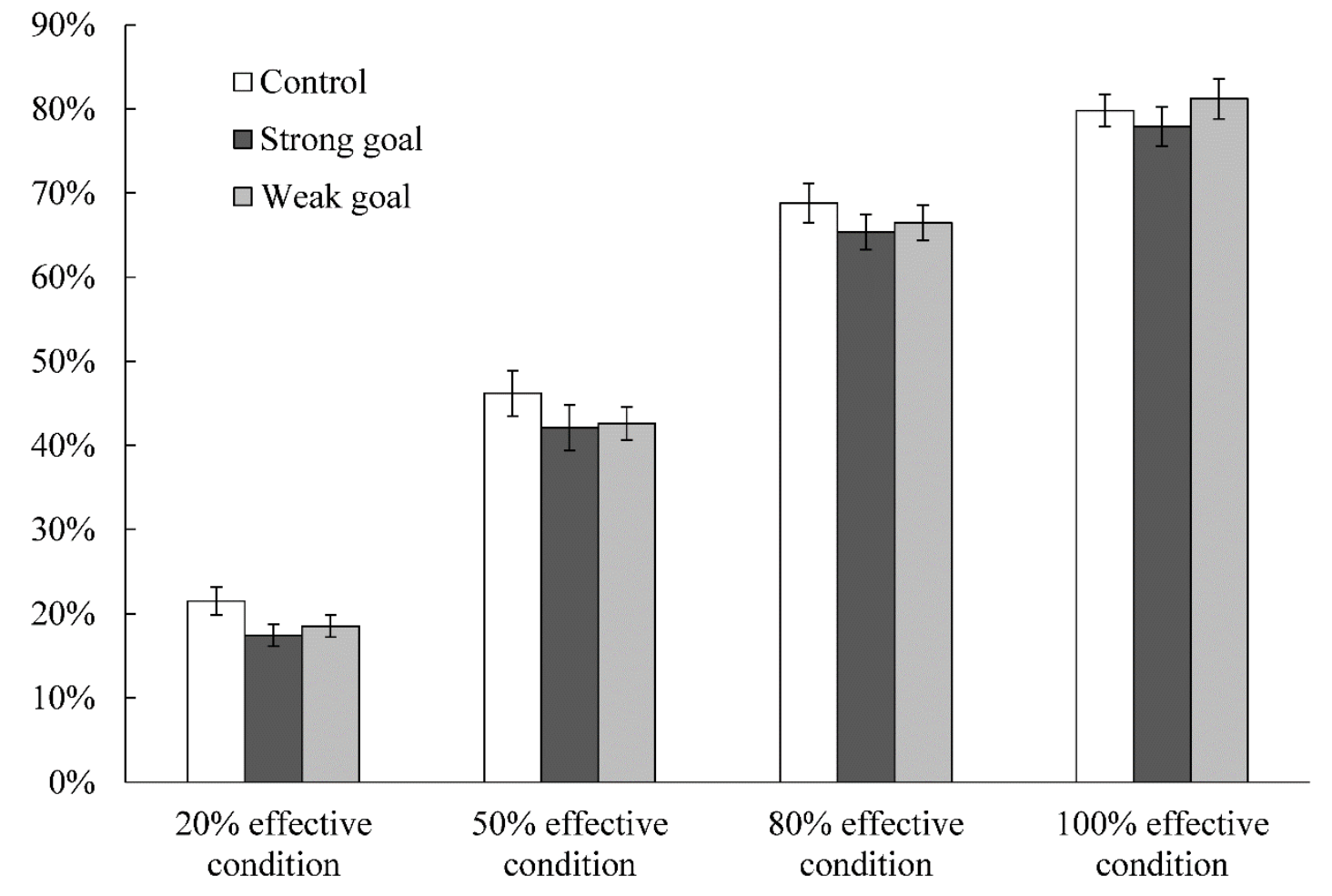

Figure 2. Mean ratings for sense of control. Error bars represent standard errors. The rating scores decreased significantly in the strong goal condition but did not decrease in 
the weak goal condition relative to the control condition, in which there was no specific goal.

Moreover, in order to further examine whether the goals influenced the sense of control through action selection or task performance, we conducted two multivariate analyses. First, we examined whether action selection influenced the sense of control. The analysis was performed via structural equation modeling using IBM SPSS Amos 22. In the model (Figure 3), the number of key presses and standard deviation for the position of the moving dot were used as the two indices of action selection. Specifically, the number of key presses represented the number of times participants decided to take action, and the standard deviation for position represented the types of action that participants took. Besides these two indices, the two independent variables (i.e., goal and effectiveness of commands) and the main dependent variable (rating of control) were also included in the model. In the model, sense of control was influenced by all of the other variables, and the two indices of action selection were influenced by the two independent variables. The standardized coefficients for all paths are depicted in Figure 3. Nonsignificant paths are shown as broken lines and significant paths are shown as solid lines (a Bonferroni-corrected $\mathrm{p}$ value of .006 was used). Only the paths from effectiveness of commands to position of the dot (i.e., standard deviation for position of 
the moving dot) and sense of control differed significantly from 0 . In summary, the results of the analysis indicated that action selection did not exert a significant influence on the sense of control.

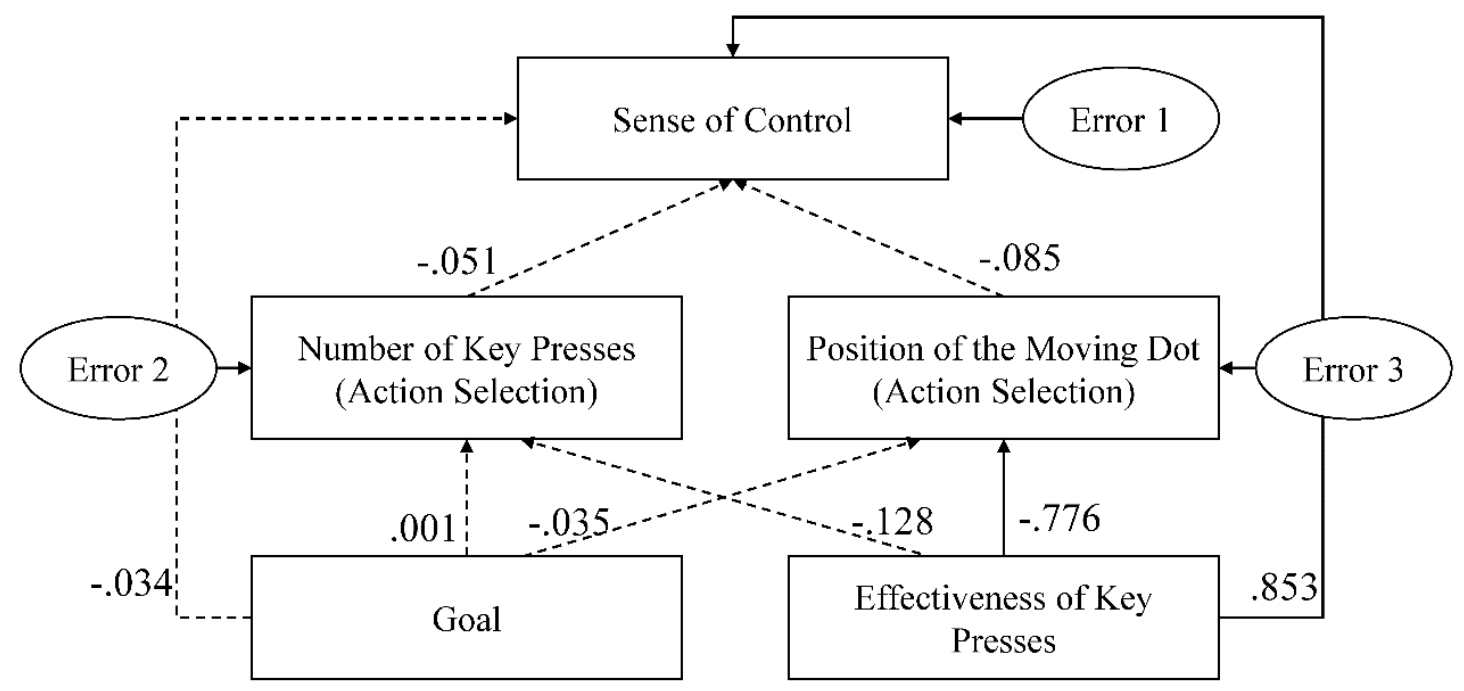

Figure 3. The structural equation model (i.e. path model) of the influence of action selection on sense of control and its predictors. The paths with significant coefficients are represented by solid lines $(p<.006)$, and the paths with nonsignificant coefficients are represented by broken lines. The model indicated that action selection did not significantly influence the sense of control. Errors 1, 2, and 3 refer to the parts that the predictors could not be accounted for.

Furthermore, we performed another multivariate analysis to examine whether task performance influenced the sense of control. This analysis was performed on the strong goal condition alone, because there was no task performance in the other two 
goal conditions. Arrival at the destination was used as the index of task performance. In the model, sense of control was influenced by both the effectiveness of key presses and task performance (Figure 4). According to the results of the analysis, all paths were significant (a Bonferroni-corrected $\mathrm{p}$ value of .017 was used). Therefore, when there was a goal accompanied by performance feedback, performance exerted a significant influence on the sense of control. That is, if performance feedback did not meet expectations, it would probably impair the sense of control. Considering the results of the two multivariate analyses in combination, we concluded that goals influenced the sense of control via goal-based expectation rather than goal-directed action selection.

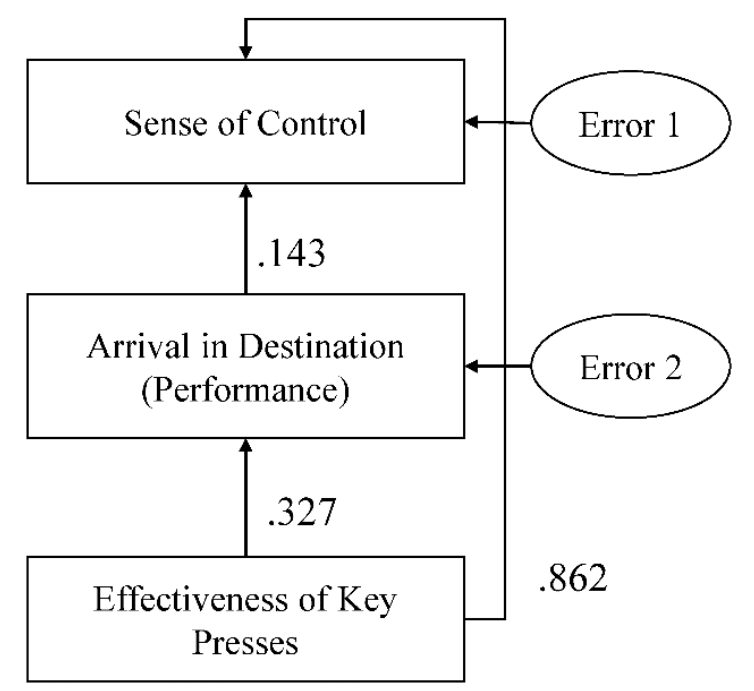

Figure 4. The structural equation model (i.e., path model) and its predictors for the influence of performance on sense of control in the strong goal condition. The coefficients of the paths were all significant $(p s<.017)$. The model indicated that task 
performance significantly influenced sense of control. Errors 1 and 2 refer to the parts that the predictors could not be accounted for.

\section{Discussion}

The purpose of the present experiment was to examine the influence of goals on the sense of control. We hypothesized that goal-directed action selection and goal-based expectation would influence sense of control. We designed two types of goal: In the strong goal condition, participants were instructed to take action according to a specific goal and received visual feedback regarding their performance regardless of whether they achieved the goal; in the weak goal condition, participants took action according to a similar goal, but the achievement of the goal was not defined clearly. Goal-directed action selection was involved in both goal conditions, but goal-based expectation was only activated in the strong goal condition. We compared the sense of control for the two goal conditions with that of a control condition, in which participants took action at will. The results showed that the sense of control decreased in the strong goal condition but did not decrease in the weak goal condition. Therefore, goal-based expectation negatively influenced the sense of control, but goal-directed action selection did not exert an influence. These findings were supported by the results of the multivariate analyses. 
To our knowledge, the present study provided the first direct evidence that the existence of goals impaired the sense of control in certain conditions. In daily life, people usually make decisions regarding their actions in order to achieve a specific goal. In such cases, intention to achieve a goal is activated. This intention is entitled distal intention and could differ from intention to initiate action (i.e., proximal intention) or intention to recall motor representation (i.e., motor intention; Pacherie, 2008). The influence of proximal and motor intention on the sense of control has been discussed in previous research (e.g., voluntary vs. involuntary actions; Haggard, Clark, \& Kalogeras, 2002; Moore, Wegner, \& Haggard, 2009; Tsakiris \& Haggard, 2005). However, distal intention has received less attention. In a recent study (Vinding, Pedersen, \& Overgaard, 2013), distal intention was reported to enhance the intentional binding effect, which is considered an implicit measure of the sense of agency (Moore \& Obhi, 2012). However, in Vinding et al.'s (2013) study, distal intention did not involve an overarching goal.

Distal intention involving an overarching goal would probably lead to two important consequences for human action and subjective feeling. First, it could influence subsequent action selection processes. Second, it probably evokes expectation of positive outcome (i.e., achievement of the goal). Both of these factors could influence the sense of control. In order to examine them, we used two types of goal. The strong 
goal was linked to clear achievement status, while the weak goal was not. The results showed that the strong goal impaired the sense of control, while weak goal did not. That is, outcome influenced the judgment of one's control, but "forced" action selection did not. Wenke et al. found that actions that were consistent with primes induced improved sense of control over action effects relative to that observed when actions were inconsistent with primes, particularly when participants were free to choose and their actions were not forced (Wenke et al., 2010). However, in the present study, we observed decrements in sense of control in the strong goal condition but not the weak goal condition, indicating that action selection did not play a role in the sense of control in our experiment. In fact, although action selection was considered to have been influenced by distal intention in both the strong and weak goal conditions, the goal did not significantly influence the number of key presses or distribution of the moving dot (Figure 3). This is likely to be because, even when there was a distal intention (and this was forced), participants generated motor intention themselves, as opposed to receiving instructions on actions as in the cued-choice trials in Wenke et al.'s (2010) experiment.

Furthermore, when distal intention exists, it not only influences motor intention but also generates a goal state, which is compared to the outcome (usually refers to task performance). Metcalfe and colleagues examined the influence of outcome on the sense 
of control and found that it plays an important role in the judgment of control (Metcalfe \& Greene, 2007; Metcalfe, Van Snellenberg, DeRosse, Balsam, \& Malhotra, 2012; Metcalfe, Eich, \& Miele, 2013). In Metcalfe et al.'s experiments, participants played a computer game in which they moved a cursor, using a mouse to touch or explode scrolling Xs. When a number of touched Xs did not explode, participants' judgment of control decreased significantly relative that observed in the condition in which all touched Xs exploded, even when participants received explicit instructions stating that they should be concerned with touching, rather than exploding Xs (Metcalfe et al., 2013). Metcalfe et al. concluded that the explosion of the Xs influenced participants' judgment of performance with respect to the number of Xs they had touched successfully, and their judgment of performance significantly influenced their judgment of control, independently of the influence of external turbulence in the cursor. However, in Metcalfe et al.'s experiment, control of the cursor was always effective, and the participants were always in perfect control in the no-turbulence condition; therefore, participants may have focused on their performance when forming judgments of control. In contrast, in the present experiment, participants' commands were not always effective (manipulated at four levels: 20\%, 50\%, 80\%, and 100\%), and they were explicitly instructed to form a judgment of control according to whether their commands had been 
successful. The results showed that the comparison of goal and outcome influenced the judgment of control, even when participants were instructed to focus on the comparison of motor commands.

In order to account for the roles of two types of comparison (action-effects and goal-outcome) in the sense of control in a systemic manner, we proposed a modified comparator model to account for distal intention (Figure 5). The comparator model provides a strong account of motor signals' effects on the sense of control (Frith et al., 2000). However, in the comparator model, predicted sensory feedback is based on motor signals. It fails to explain the illusionary sense of control when no motor signal exists (e.g, Wegner et al., 2004). Recently, Carruthers reviewed and defended this model with the common objection that actual sensory consequences of action are unnecessary in the sense of agency (Carruthers, 2012). However, without extensive modification, it remains difficult to explain the results of postdictive inferences of agency using the comparator model. The proposed model could account for the results of the present study and Metcalfe et al.'s $(2007,2012,2013)$ findings and produce an integrated account of predictive and postdictive processes in the sense of control. 


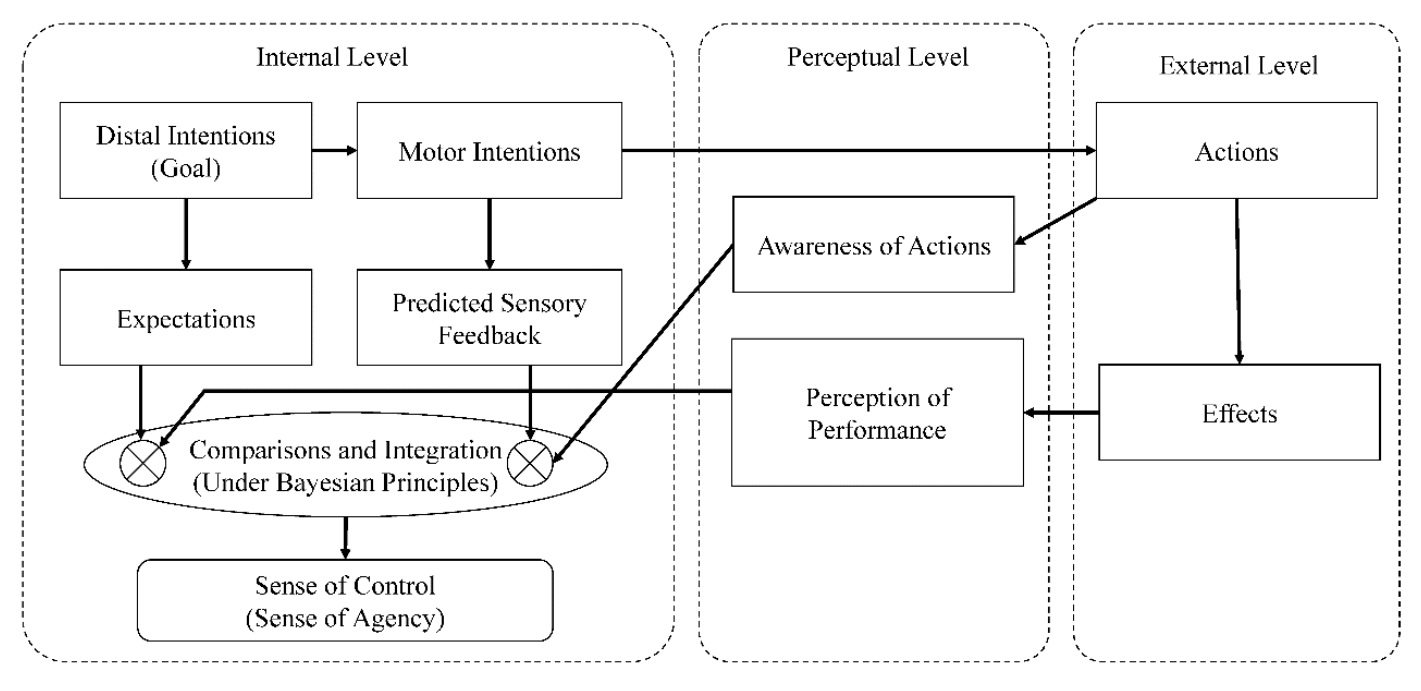

Figure 5. A modified comparator model of sense of control. The model includes three levels: internal, perceptual, and external. During an action, distal intention and motor intention are generated and produce two difference pathways for comparison. First, expectations are generated via distal intention and compared with the perception of performance. Second, similar to the original comparator model, predictions are generated via motor intention and awareness of action and compared with actual sensory feedback. The results of the two comparisons are integrated under Bayesian principles and generate the sense of control.

There were three levels in our model: internal, perceptual, and external. The internal level refers what occurs in the mind. The perceptual level refers to sensory processes, such as visual, audio, and bodily sensations. The external level refers to physical interaction with the environment. There are three points of note regarding our model. First, both distal and motor intention are included in the model. Perception of 
action and performance is compared with predicted sensory feedback, which is generated via motor intention and awareness of action in a similar manner to that of the original comparator model and prediction (i.e., expectation) based on distal intention, respectively. Second, these two types of comparison are integrated, generally under Bayesian principles, as suggested by cue integration theory (Moore \& Fletcher, 2012) and optimal cue integration theory (Synofzik, Vosgerau, \& Voss, 2013). In the present study, although the participants were explicitly instructed to form a judgment of the action-effect comparison, the goal-outcome comparison affected their judgment, indicating that the latter type of process is strong and could involve implicit factors. Third, we replaced motor signals in the original model with intention to act and awareness of action. A neuroscience study using a direct electric stimulus on the cortex revealed that intention to move and awareness of movement are represented in different brain regions and distinct from each other (Desmurget et al., 2009). Therefore, intention to act and awareness of action should be distinguished in the model of the sense of control. Furthermore, motor commands alone could be insufficient for the generation of the sense of control. For example, a previous study reported that two patients who had lost their senses of proprioception and touch retained the motor abilities required to observe their movements, in order to experience a sense of agency (Cole \& Paillard, 
1995). In this case, motor signals were insufficient for the generation of the sense of agency. Rather, awareness of action was critical.

The proposed model provides a strong explanation for our results. The existence of a strong goal probably influenced the expectation generated via distal intention. When actual sensory feedback did not meet expectations, sense of control decreased. With a weak goal, because strict expectations were not generated and performance feedback was not sensed, comparisons based on distal intentions were not activated and did not influence the sense of control. In addition, motor intention may have differed between the two goal conditions and the control condition. However, because the actual sensory feedback also changed accordingly, the results of the comparisons between them probably did not fluctuate extensively.

The proposed model accounted for not only predictive processes, as the original comparator model does, but also postdictive processes. For example, in Wegner and colleagues' helping hand paradigm (2004), the pathway from motor intention to act and awareness of action and the correspondent comparison were not activated. However, when the participants heard instructions for the movement in advance, the pathway from distal intention to expectation and the correspondent comparisons were probably activated and therefore produced the sense of agency. When the pathway of action is 
absent, the pathway based on distal intention is similar to the illusion of "mind control," in which one might feel that one is able to cause external effects with one's mind without performing any actual action.

In conclusion, the present study provided direct evidence that the existence of a goal influences the sense of control. Existence of a goal could impair the sense of control if actual sensory feedback does not match expectations based on the goal. Furthermore, we proposed a modified comparator model to account for the sense of control, including a pathway of comparison between distal intention (i.e., the goal) and actual sensory feedback. In addition, we replaced motor commands with motor intention and awareness of action, to enable the comparator model to account for cases in which motor commands are insufficient (e.g., Cole \& Paillard, 1995). The proposed model offers a promising approach to the integration of the predictive and postdictive processes involved in the sense of control.

\section{Acknowledgments}

This work was supported in part by the Sasakawa Scientific Research Grant from the Japanese Science Society, JSPS KAKENHI Grant Number 15K16007, 26120005, and the JST RISTEX Service Science, Solutions, and Foundation Integrated Research Program. 


\section{References}

Aarts, H., Custers, R., \& Wegner, D. M. (2005). On the inference of personal authorship: Enhancing experienced agency by priming effect information. Consciousness and Cognition, 14(3), 439-58. doi:10.1016/j.concog.2004.11.001

Blakemore, S.-J., Frith, C. D., \& Wolpert, D. M. (1999). Spatio-temporal prediction modulates the perception of self-produced stimuli. Journal of Cognitive Neuroscience, 11(5), 551-559. doi:10.1162/089892999563607

Blakemore, S.-J., Wolpert, D. M., \& Frith, C. D. (1998). Central cancellation of self-produced tickle sensation. Nature Neuroscience, 1(7), 635-640.

Blakemore, S.-J., Wolpert, D. M., \& Frith, C. D. (2002). Abnormalities in the awareness of action. Trends in Cognitive Sciences, 6(6), 237-242. doi:10.1016/S1364-6613(02)01907-1

Carruthers, G. (2012). The case for the comparator model as an explanation of the sense of agency and its breakdowns. Consciousness and Cognition, 21(1), 30-45. doi:10.1016/j.concog.2010.08.005

Cole, J., \& Paillard, J. (1995). Living without touch and peripheral information about body position and movement: Studies with deafferented subjects. In J. L. Bermudez, A. Marcel, \& N. Eilan (Eds.), The body and the self. MIT.

Desmurget, M., Reilly, K. T., Richard, N., Szathmari, a., Mottolese, C., \& Sirigu, a. (2009). Movement Intention After Parietal Cortex Stimulation in Humans. Science, 324(5928), 811-813. doi:10.1126/science.1169896

Frith, C. D., Blakemore, S.-J., \& Wolpert, D. M. (2000). Explaining the symptoms of schizophrenia $\square$ : Abnormalities in the awareness of action. Brain Research Reviews, 31, 357-363.

Haggard, P., Clark, S., \& Kalogeras, J. (2002). Voluntary action and conscious awareness. Nature Neuroscience, 5(4), 382-385. doi:10.1038/nn827 
Metcalfe, J., Eich, T. S., \& Miele, D. B. (2013). Metacognition of agency: Proximal action and distal outcome. Experimental Brain Research, 229(3), 485-496. doi:10.1007/s00221-012-3371-6

Metcalfe, J., \& Greene, M. J. (2007). Metacognition of agency. Journal of Experimental Psychology. General, 136(2), 184-199. doi:10.1037/0096-3445.136.2.184

Metcalfe, J., Van Snellenberg, J. X., DeRosse, P., Balsam, P., \& Malhotra, a. K. (2012). Judgements of agency in schizophrenia: an impairment in autonoetic metacognition. Philosophical Transactions of the Royal Society B: Biological Sciences, 367(1594), 1391-1400. doi:10.1098/rstb.2012.0006

Moore, J. W., \& Fletcher, P. C. (2012). Sense of agency in health and disease: A review of cue integration approaches. Consciousness and Cognition, 21(1), 59-68. doi:10.1016/j.concog.2011.08.010

Moore, J. W., \& Obhi, S. S. (2012). Intentional binding and the sense of agency: A review. Consciousness and Cognition, 21(1), 546-61. doi:10.1016/j.concog.2011.12.002

Moore, J. W., Wegner, D. M., \& Haggard, P. (2009). Modulating the sense of agency with external cues. Consciousness and Cognition, 18(4), 1056-64. doi:10.1016/j.concog.2009.05.004

Pacherie, E. (2008). The phenomenology of action: A conceptual framework. Cognition, 107(1), 179-217. doi:10.1016/j.cognition.2007.09.003

Synofzik, M., Vosgerau, G., \& Newen, A. (2008). Beyond the comparator model: A multifactorial two-step account of agency. Consciousness and Cognition, 17(1), 219-239. doi:10.1016/j.concog.2007.03.010

Synofzik, M., Vosgerau, G., \& Voss, M. (2013). The experience of agency: An interplay between prediction and postdiction. Frontiers in Psychology, 4(MAR), 1-8. doi:10.3389/fpsyg.2013.00127

Tsakiris, M., \& Haggard, P. (2005). Experimenting with the acting self. Cognitive Neuropsychology, 22(3), 387-407. doi:10.1080/02643290442000158 
Van der Weiden, A., Ruys, K. I., \& Aarts, H. (2013). A matter of matching: How goals and primes affect self-agency experiences. Journal of Experimental Psychology. General, 142(3), 954-66. doi:10.1037/a0030079

Vinding, M. C., Pedersen, M. N., \& Overgaard, M. (2013). Unravelling intention: Distal intentions increase the subjective sense of agency. Consciousness and Cognition, 22(3), 810-815. doi:10.1016/j.concog.2013.05.003

Wegner, D. M. (2002). The illusion of conscious will. Cambridge MA: MIT Press.

Wegner, D. M. (2003). The mind's best trick: How we experience conscious will. Trends in Cognitive Sciences, 7(2), 65-69. doi:10.1016/S1364-6613(03)00002-0

Wegner, D. M., Sparrow, B., \& Winerman, L. (2004). Vicarious agency: Experiencing control over the movements of others. Journal of Personality and Social Psychology, 86(6), 838-848. doi:10.1037/0022-3514.86.6.838

Wegner, D. M., \& Wheatley, T. (1999). Apparent mental causation: Sources of the experience of will. American Psychologist, 54(7), 480-492.

Wen, W., Yamashita, A., \& Asama, H. (2015). The sense of agency during continuous action: Performance is more important than action-feedback association. PLoS ONE, 10(4), e0125226. doi:10.1371/journal.pone.0125226

Wenke, D., Fleming, S. M., \& Haggard, P. (2010). Subliminal priming of actions influences sense of control over effects of action. Cognition, 115(1), 26-38. doi:10.1016/j.cognition.2009.10.016

Wolpert, D. M., \& Flanagan, J. R. (2001). Motor prediction. Current Biology, 11(18), R729-R732.

Wolpert, D. M., \& Ghahramani, Z. (2000). Computational principles of movement neuroscience. Nature Neuroscience, 3(Suppl.), 1212-1217. 